Pensamiento Crítico $\mathrm{N}^{\circ} 16$, pp. 7-19

\title{
Análisis de la gestión del riesgo de la banca múltiple en el Perú: 2000-2010
}

Mg. Gaby Cortez Cortez

\section{RESUMEN}

El trabajo busca analizar la gestión del riesgo de los bancos que realizan sus operaciones en el Perú, mediante la aplicación de indicadores que relacionan el rendimiento de las operaciones con el riesgo. El RAROC (Risk Adjusted Return on Capital o Rentabilidad del Capital Ajustada por el Riesgo) es uno de estos indicadores que asocia los ingresos netos de una operación o conjunto de operaciones del banco en un año sobre el capital de riesgo o capital económico. La versatilidad de este indicador permite medir la gestión del riesgo del banco de manera transversal, comparándolo con otros bancos o con un benchmark del mercado, así como también medir la evolución de la gestión del riesgo a través de un periodo de tiempo comparándolo con un RAROC objetivo o meta.

Los resultados muestran que los bancos grandes revelaron un RAROC por encima del promedio del mercado, 2.07 vs. 1.75. Los bancos medianos mostraron un RAROC por debajo del promedio general: 1.23 vs. 1.75 . Finalmente, los bancos pequeños obtuvieron un ratio promedio del periodo de 1.74 vs. 1.75 , sin embargo, al interior del periodo mostraron un comportamiento fluctuante, sobre y por debajo del promedio general.

Palabras clave: riesgo bancario, gestión de riesgo, RAROC. 


\title{
Pensamiento Crítico $\mathrm{N}^{\circ} 16$
}

\begin{abstract}
The paper analyzes the risk management of banks that do business in Peru, through the application of indicators that relate the profitability of transactions with the risk. The RAROC (Risk adjusted Return on Capital) is one of the indicators associated with net income of an operation or set of operations of the bank in a year for capital at risk o economic capital. The versatility of this indicator allows to measure the bank 's risk management in a cross section, compared to other banks or a market benchmark, as well as to measure the evolution of risk management through a period of time compared to a RAROC target.

The result shows that the Big Banks revealed a RAROC above the market average: 2.07 vs. 1.75. Medium Banks showed a RAROC below the overall average: 1.23 vs. 1.75. Finally Small Banks obtained an average ratio of 1.74 vs. 1.75 , however within the period they showed a fluctuating behavior, above and below the average.
\end{abstract}

Keywords: bank risk, risk management, RAROC.

\section{Introducción}

La participación de un banco en el mercado se basa en un principio esencial: su capital tiene que redituar por lo menos una rentabilidad igual, o en el mejor de los casos, por encima del promedio del mercado.

Si este objetivo no se cumple, el banco evalúa su participación, buscando mejorar la relación de riesgo y rendimiento de su inversión.

Así de sencilla es la racionalidad empresarial, de lo contrario, el banco reduce su posición en el mercado o en el peor de los casos, se ve obligado a salir del mercado, es decir puede quebrar.

Con el objetivo de mantenerse en el mercado, los bancos y los profesionales dedicados al análisis de la gestión empresarial buscan comprender los factores que afectan la relación entre riesgo-rendimiento.

Para tal efecto, se construyen indicadores para medir tanto el desempeño del banco como dicha relación. 


\section{Gaby Cortez Cortez}

Uno de estos indicadores, quizás el más importante para el análisis de la rentabilidad en finanzas, es el ROE (Return on Equity) o rentabilidad del capital. En base a este ratio, se han construido indicadores que sirven para el análisis de la relación riesgo-rendimiento, como es el caso del RAROC (Risk Adjusted Return on Capital) o rentabilidad del capital ajustada por el riesgo.

\section{Revisión de la literatura}

El trabajo de Gene Guill, "Bankers Trust and the Birth of Modern Risk Management" (2009), señala que Bankers Trust reconoció que el viejo paradigma de la banca comercial como el intermediario que recoge los depósitos y otorga préstamos, era un negocio en declive y define un nuevo modelo de negocio para la banca moderna. Sostiene además, que la desregulación, la competencia global, el desarrollo de nuevos productos, y los cambios tecnológicos introducen un mayor riesgo en el negocio de la banca. Con el fin de sobrevivir en este nuevo mundo, los bancos tendrían que aprender a manejar el riesgo.

La estrategia que guía la transformación según Bankers Trust se centró en la gestión del riesgo, la implementación productiva del capital, el desarrollo de nuevos productos y el desarrollo de nuevos mercados. Se deja de lado la adquisición de competidores o la acumulación agresiva de activos.

El estudio sostiene que los bancos de éxito están en el negocio de la gestión del riesgo, tanto como lo están en el negocio de la gestión de rentabilidad. Si una empresa entiende el riesgo, puede llevar a cabo lo siguiente: a) tomar decisiones conscientes para aprovechar o deshacerse de riesgos, b) cobrar un precio racional por el riesgo que se asume; c) redistribuir el capital de las actividades de bajo rendimiento a las que ganan rentabilidades ajustadas al riesgo en exceso de un objetivo determinado; y d) juzgar con precisión la cantidad total de capital que se necesita para mantener como un amortiguador frente a las pérdidas imprevistas.

El estudio de Sinan Cebenoyan, Philip E. Strahan, "Risk management, capital structure and lending at Banks" (2004), comprueba cómo la gestión de la exposición al riesgo de crédito bancario a través del mercado de ventas de préstamos afecta a la estructura de capital, a los préstamos, a los beneficios y a los riesgos de un banco. 


\section{Pensamiento Crítico $\mathrm{N}^{\circ}$ I6}

Los autores encuentran que los bancos que buscan equilibrar la exposición de su portafolio de préstamos, tanto mediante la compra y venta de préstamos -es decir, los bancos que utilizan el mercado de ventas de préstamos para propósitos de la gestión del riesgo, en vez de modificar sus tenencias de préstamos- mantienen menos capital que otros bancos.

Estos bancos hacen préstamos más arriesgados que otros, como un porcentaje del total de activos. El tamaño de los préstamos, el apalancamiento y los préstamos constantes hacen que los bancos que dinámicos en el mercado de ventas de préstamos tengan un menor riesgo y mayores beneficios que otros bancos.

Los resultados que encuentran sugieren que los bancos que mejoran su capacidad para gestionar el riesgo de crédito, puedan operar con mayor apalancamiento y prestar más de sus activos a prestatarios riesgosos. Así, los beneficios de los avances en la gestión de riesgos en la banca pueden traducirse en una mayor disponibilidad de crédito, en lugar de reducir el riesgo del sistema bancario.

De otro lado, la investigación de Hsien-Hsing Liao, Tsung - Kang Chen, Chia - Wu Lu, "Riesgo de crédito bancario y modelos estructurales de crédito: Perspectivas de la Agencia e información asimétrica", nos muestra los efectos de la teoría de la agencia y la asimetría de la información integradas en las formas estructurales de los modelos de crédito, para la evaluación del riesgo de crédito de los bancos, utilizando los datos de bancos estadounidense desde el 2001 hasta 2005.

Los resultados que encuentran los investigadores muestran que tanto el problema de la agencia y la asimetría de la información causan desviaciones significativas en la evaluación del riesgo de crédito de los modelos de forma estructural en relación a los modelos de las agencias de calificación. Se encuentran cinco factores independientes que explican una desviación de $42,6 \%$ a 78,3\%, y que deben incorporarse en el futuro en el modelo de riesgo de crédito. Además, tanto los efectos de la asimetría de la información y el problema de la agencia sobre deuda-capital, están relacionados positivamente con esta desviación, mientras que el problema de la agencia de gestión-capital se relaciona con el problema de la agencia de manera negativa. 


\section{Gaby Cortez Cortez}

\section{Problema}

Se observa que durante el periodo de análisis hubo un cambio en la estructura por tipo de crédito, disminuyendo la participación porcentual de las colocaciones al comercio y a la microempresa, mientras que, el crédito hipotecario y de consumo tuvieron un aumento. Este cambio obedecería a la búsqueda del mayor beneficio, que está a su vez vinculado a un mayor riesgo.

Se parte de la premisa que una mayor concentración de créditos en determinado sector económico, aumenta el riesgo de las operaciones de un banco, y una mayor diversificación disminuye la posibilidad de riesgo.

Los bancos que no pudieron diversificar adecuadamente su cartera de préstamos, asumieron un mayor riesgo sin encontrar una mayor rentabilidad, generando su salida del mercado. Un objetivo principal de los gerentes de los bancos es aumentar la rentabilidad de los propietarios. Sin embargo, esto casi siempre viene acompañado del incremento del riesgo de las operaciones de los bancos.

Este planteamiento nos lleva a formular la siguiente interrogante:

\section{¿Por qué los bancos ante situaciones similares de riesgo tienen respuestas diferentes en la gestión del mismo?}

\section{Hipótesis general}

Los cambios en la estructura de la cartera de créditos de los bancos, así como en la diversificación de las operaciones, conducen a mejorar la rentabilidad disminuyendo la posibilidad de riesgo.

\section{Hipótesis secundaria}

El RAROC (Rentabilidad del capital ajustada por el riesgo) permite evaluar la gestión del riesgo de los bancos, tanto en su desempeño horizontal; es decir, compararlo con otros bancos o con un benchmark del mercado, así como en su desempeño vertical; es decir, a través de un horizonte temporal observando su desempeño en el tiempo y cambios realizados en la estructura y tipos de crédito. 


\section{Pensamiento Crítico $\mathrm{N}^{\circ} 16$}

\section{Métodos y resultados}

Debido a que las estadísticas que publica la Superintendencia de Banca y Seguros sobre capital de riesgo (por tipo de riesgo) son de reciente publicación y el periodo de análisis del trabajo es del 2000-2010, se ha utilizado una variante del RAROC que relaciona el rendimiento promedio del ROE (Return on Equity o Rentabilidad del Capital) en un año sobre la desviación estándar del promedio del ROE como medida de la volatilidad o riesgo.

Para el trabajo se ha escogido una muestra de bancos que han operado en la totalidad del periodo (excepto por el Banco del Trabajo que no llegó a culminar su permanencia hasta el final del periodo). Se agruparon los bancos de acuerdo a la magnitud de sus activos, formando grupos de bancos grandes, medianos y pequeños.

Se utilizó el modelo RAROC ( Risk- adjusted return on Capital) o el modelo de rentabilidad del capital ajustada por el riesgo.

RAROC es una medida de rentabilidad que puede aplicarse a operaciones aisladas o a una cartera de préstamos. Es una medida que también incorpora los riesgos que se asumen al realizar una operación de crédito. Es un indicador para la gestión del negocio bancario que mide la relación entre rentabilidad y la gestión del riesgo.

$\mathrm{RAROC}=$ Utilidad neta $/$ Capital en riesgo

El análisis del RAROC revela la cantidad de capital económico que es requerido por cada línea de negocio, producto o cliente, y cómo estos requerimientos crean la rentabilidad total de capital producido por la empresa. La Superintendencia de Banca y Seguros del Perú recién ha empezado a trabajar el capital ajustado por el riesgo, a partir de agosto 2009, por lo que no se cuenta con la información para todo el periodo de análisis.

En tal sentido, para suplir esta deficiencia y a fin de realizar un análisis de largo plazo se ha usado el siguiente indicador: 


\section{Gaby Cortez Cortez}

$\mathrm{RAROC}=$ Promedio anual de ROE / Promedio anual de la desviación estándar del ROE.

En donde:

- El numerador mide la rentabilidad del capital.

- El denominador mide la volatilidad de la rentabilidad o riesgo.

Los resultados encontrados se resumen en el Cuadro 1.

Cuadro 1 RAROC DE LA BANCA MULTIPLE: 2000-2010

\begin{tabular}{|c|c|c|c|c|c|c|c|c|c|c|c|c|}
\hline & \multicolumn{3}{|c|}{ BANCOS GRANDES } & \multicolumn{3}{|c|}{ BANCOS MEDIANOS } & \multicolumn{3}{|c|}{ BANCOS PEQUEñOS } & \multicolumn{3}{|c|}{ BANCA MULTIPLE } \\
\hline & Media & D. E. & RAROC & Media & D. E. & RAROC & Media & D. E. & RAROC & Media & D. E. & RAROC \\
\hline & ROE & ROE & & ROE & ROE & & ROE & ROE & & ROE & ROE & \\
\hline 2000 & 2.20 & 1.10 & 2.00 & -0.74 & 2.22 & -0.33 & 10.26 & 4.96 & 2.07 & 2.38 & 1.25 & 1.91 \\
\hline 2001 & 3.68 & 2.80 & 1.31 & -1.51 & 2.32 & -0.65 & 8.08 & 4.48 & 1.80 & 3.73 & 2.50 & 1.53 \\
\hline 2002 & 9.28 & 3.90 & 2.38 & 0.24 & 3.80 & 0.06 & 19.47 & 12.89 & 1.51 & 7.12 & 3.21 & 2.22 \\
\hline 2003 & 10.70 & 5.63 & 1.90 & 3.90 & 2.21 & 1.77 & 23.40 & 13.34 & 1.75 & 7.64 & 5.29 & 1.44 \\
\hline 2004 & 12.58 & 5.68 & 2.22 & 3.33 & 2.11 & 1.57 & 21.90 & 12.08 & 1.81 & 9.43 & 5.33 & 1.77 \\
\hline 2005 & 23.90 & 12.80 & 1.87 & 7.35 & 4.49 & 1.64 & 25.52 & 14.45 & 1.77 & 16.61 & 12.56 & 1.32 \\
\hline 2006 & 31.55 & 16.08 & 1.96 & 6.94 & 4.65 & 1.49 & 18.31 & 9.08 & 2.02 & 21.66 & 13.20 & 1.64 \\
\hline 2007 & 43.98 & 21.58 & 2.04 & 7.76 & 5.41 & 1.43 & 15.34 & 10.80 & 1.42 & 30.06 & 16.48 & 1.82 \\
\hline 2008 & 48.20 & 23.55 & 2.05 & 9.47 & 5.28 & 1.79 & 17.45 & 10.43 & 1.67 & 32.86 & 18 & 1.83 \\
\hline 2009 & 43.60 & 19.25 & 2.26 & 7.16 & 4.64 & 1.54 & 24.70 & 15.20 & 1.63 & 25.45 & 12.34 & 2.06 \\
\hline 2010 & 34.08 & 15.20 & 2.24 & 7.48 & 4.72 & 1.58 & 23.50 & 11.77 & 2.00 & 21.61 & 12.34 & 1.75 \\
\hline Prom & 23.98 & 11.60 & 2.07 & 4.67 & 3.80 & 1.23 & 18.90 & 10.86 & 1.74 & 16.23 & 9.32 & 1.75 \\
\hline
\end{tabular}

\section{RAROC de la Banca Grande}

Se ha incluido en la Banca Grande a los bancos Continental - BBVA, Banco de Crédito, Scotiabank e Interbank.

Los resultados encontrados (Ver Cuadro 1 y Gráfico 1) nos muestran que del 2002 al 2010 los bancos grandes tuvieron un RAROC por encima del RAROC del mercado, indicándonos de esta manera que el manejo del riesgo de estos bancos les permitió obtener una relación riesgo-rendimiento superior al promedio del sistema. A su vez, nos señala que ha existido un mejor conocimiento de la gestión de productos y servicios puestos a disposición de sus clientes. 
Gráfico 1.

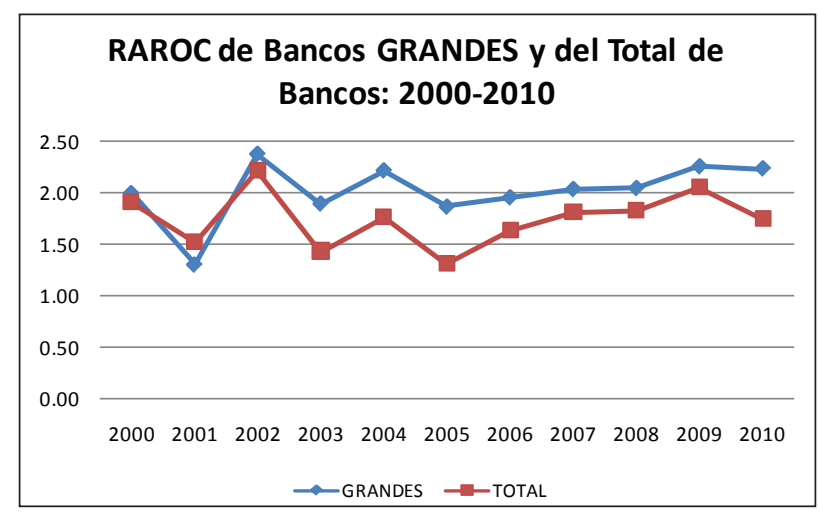

De otro lado, al analizar los componentes del RAROC de la banca grande se encuentra que:

1. Se produjo un proceso de reajuste del 2000 al 2004, con un ligero aumento de la rentabilidad, vinculado a un menor riesgo (Ver Gráfico 2).

2. Se observa un aumento de la rentabilidad del 2005 al 2008, asociado a un aumento del riesgo, pero en menor grado, que corresponde a un RAROC mayor que el del mercado.

3. Disminuyó la rentabilidad del 2009 al 2010, asociada a una reducción del riesgo y a un RAROC todavía por encima del mercado.

Gráfico 2.

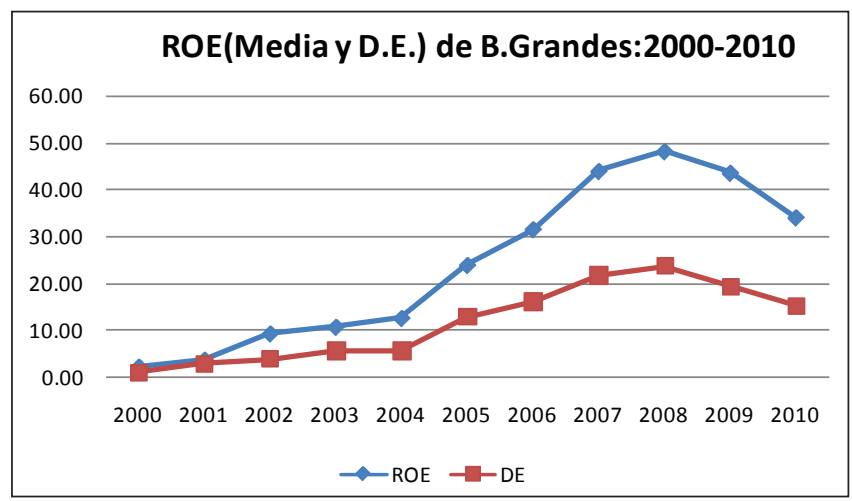




\section{Gaby Cortez Cortez}

Estos resultados señalan que los bancos grandes han gestionado su cartera de préstamos de forma tal que la diversificación en distintos sectores económicos les ha permitido tener una relación riesgo-rendimiento superior al sistema.

\section{RAROC de la Banca Mediana}

En la Banca Mediana se ha incluido a los bancos Financiero, Banco de Comercio e Interamericano de Finanzas.

Los resultados encontrados (Ver Cuadro 1 y Gráfico 3) nos muestran que del 2000 al 2002 los bancos medianos tuvieron un RAROC por debajo del RAROC del mercado. Debe mencionarse que durante estos años, varios de estos bancos estuvieron tratando de mantenerse en el sistema, pero finalmente tuvieron que salir del mercado debido a problemas de alta concentración de su portafolio de préstamos en algunos sectores económicos específicos, tal como el caso de los bancos Sudamericano, Standard Chartered, Bank of Boston, BNP Paribas y Santander.

Luego de este proceso y de haber perdurado a la crisis financiera de 1998-1999, los bancos Financiero, de Comercio e Interamericano de Finanzas, obtuvieron un RAROC por debajo del promedio del sistema la mayor parte del periodo bajo análisis, lo que nos indica que la gestión de riesgo tuvo muchas dificultades para igualar o superar el promedio del mercado.

Gráfico 3.

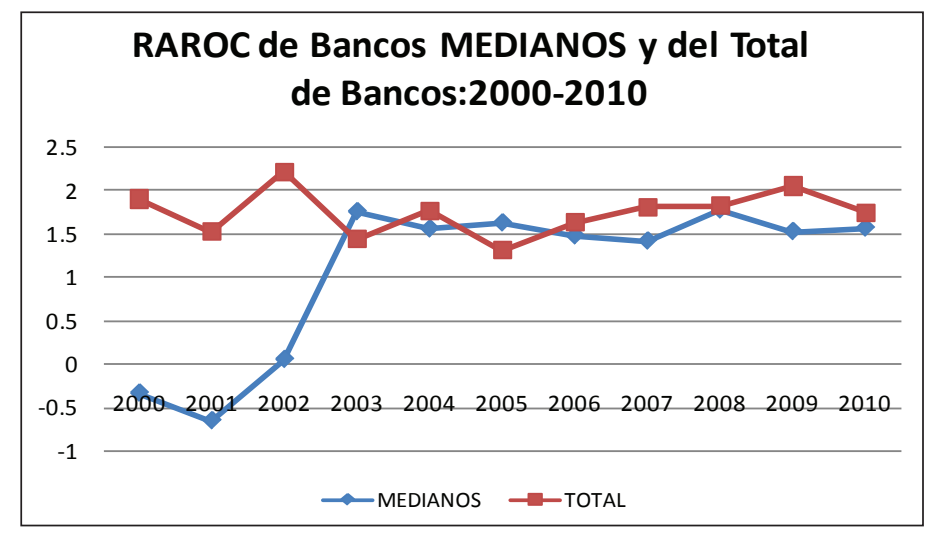




\section{Pensamiento Crítico $\mathrm{N}^{\circ} 16$}

Los componentes del RAROC de los bancos medianos muestran el siguiente comportamiento (Ver Gráfico 4):

1. Una rentabilidad negativa del 2000 al 2002, la que estuvo asociada a un mayor riesgo, con un RAROC por debajo del promedio del mercado. Estos resultados constituyen un caso especial ya que los bancos estuvieron generando pérdidas por la alta morosidad en que incurrieron.

2. Del 2003 al 2008 se restablece la relación riesgo-rentabilidad, con un RAROC oscilante con el mercado del 2003 al 2005, para luego situarse por debajo del mercado del 2006 al 2010.

Gráfico 4.

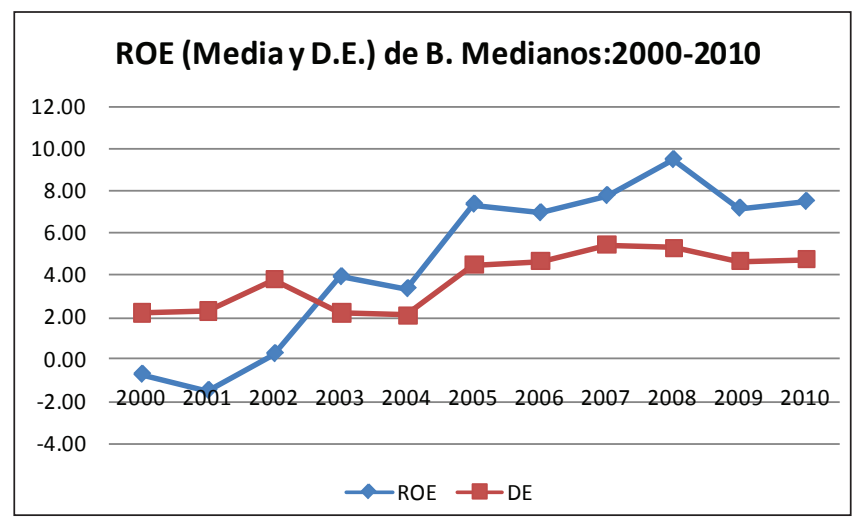

Estos resultados nos indican que los bancos medianos han tenido dificultades en la gestión del riesgo de préstamos.

\section{RAROC de la Banca Pequeña}

La banca pequeña está conformada por los bancos del Trabajo y Mibanco. Se encuentra que durante el periodo de análisis el RAROC de los bancos pequeños ha tenido un comportamiento oscilante alrededor del RAROC del mercado. Esto puede explicarse por la fragilidad de estos bancos debido a la alta concentración de su cartera de préstamos en algunos sectores económicos. (Ver el Cuadro 1 y Gráfico 5). 


\section{Gaby Cortez Cortez}

Gráfico 5.

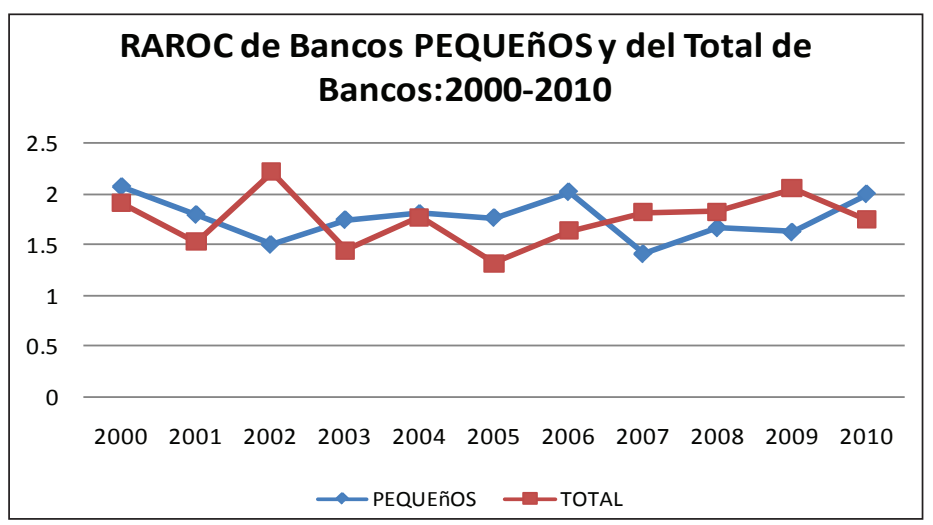

Del análisis de los componentes del RAROC de los bancos pequeños, se puede colegir lo siguiente:

1. Se mantiene la relación riesgo-rentabilidad del 2000 al 2010, tal como puede observarse en el Gráfico 6. Asimismo, se puede prestar atención al periodo del 2001 al 2005 en el que aumenta la rentabilidad de estos bancos, la que está vinculada con un aumento del riesgo, pero de manera menos que proporcional.

2. Del 2006 al 2007 disminuye la rentabilidad de los bancos pequeños, para luego, del 2008 al 2009 retomar el incremento del beneficio, siempre con el nivel de riesgo respectivo.

Gráfico 6.

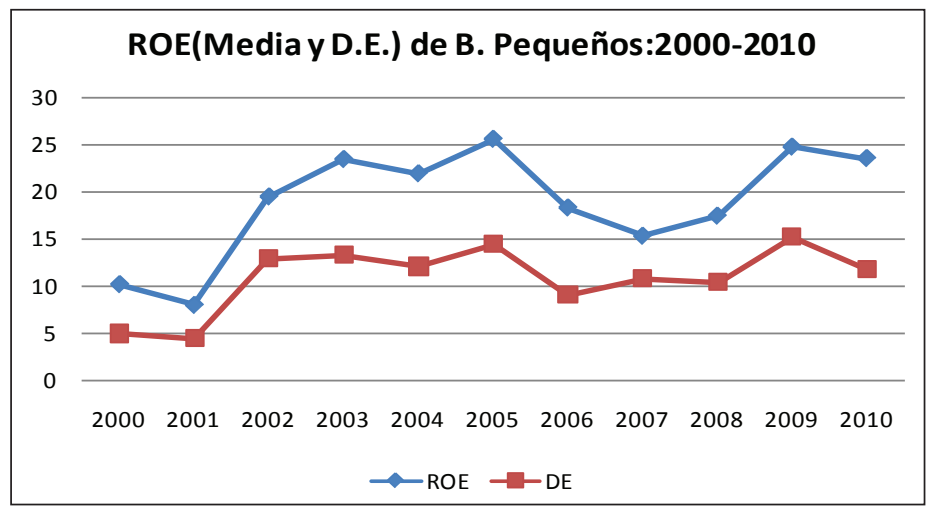




\section{Pensamiento Crítico $\mathbf{N}^{\circ} 16$}

Los bancos pequeños obtuvieron un RAROC promedio de 1.74 versus 1.75 del mercado, mostrando un comportamiento fluctuante, sobre y por debajo del promedio general, revelando los problemas que enfrentaron en la gestión del riesgo.

\section{Conclusiones}

- Los bancos grandes muestran una cartera de préstamos más variada que la de los bancos medianos y pequeños, lo cual les permite diversificar mejor el riesgo y obtener un RAROC por encima del promedio general (2.07 versus 1.75), mostrando una mejor gestión del riesgo crediticio. Los bancos medianos mostraron un RAROC (1.23) por debajo del promedio general. Finalmente, los bancos pequeños obtuvieron un RAROC (1.74) cercano al promedio del periodo; sin embargo, al interior del periodo mostraron un comportamiento fluctuante sobre y por debajo del promedio general.

- Los bancos medianos han operado en un espacio que se ha venido reduciendo por la salida de algunos bancos, los que tuvieron una alta concentración de préstamos en algún sector económico, tal como el caso de los bancos Sudamericano, Standard Chartered, Bank of Boston, BNP Paribas y Santander.

- Ha tomado cierto tiempo a los bancos de Comercio, Financiero e Interamericano de Finanzas estabilizarse, luego de la crisis financiera de 1998-1999; lo que se traduce en un RAROC de la banca mediana por debajo del promedio general la mayor parte del periodo de análisis, mostrando una gestión de riesgo crediticio con dificultades para igualar o superar el promedio del sistema.

- Los bancos pequeños que se han mantenido en la mayor parte del periodo de análisis (B. del Trabajo y Mibanco), han mostrado cierta fragilidad, principalmente por la concentración de sus operaciones, lo que conduce a un RAROC de comportamiento fluctuante, sobre y por debajo del promedio de mercado. 


\section{Gaby Cortez Cortez}

\section{Referencias bibliográficas}

Cebenoyan, Sinan y Strahan, Philip E. Risk management, capital structure and lending at Banks. Journal of Banking \& Finance, 2004.

Guill, Gene. Bankers Trust and the Birth of Modern Risk Management. Financial Institutions Center. Wharton, 2009.

Hsien-Hsing Liao, Tsung - Kang Chen, Chia - Wu Lu, Bank credit risk and structural credit models: Agency and information asymmetry perspectives, 2009. 